\title{
MAGNETIC TWO-AXIS MICROMIRROR FOR 3D OCT ENDOSCOPY
}

\author{
Jonathan J. Bernstein, Tom W. Lee, Fran J. Rogomentich, and Mirela G. Bancu \\ The Charles Stark Draper Laboratory \\ Cambridge, MA USA \\ Ki H. Kim, Gopi Maguluri, Brett E. Bouma, and Johannes F. DeBoer \\ Wellman Laboratory of Photomedecine, Massachusetts General Hospital \\ Boston, MA USA
}

\begin{abstract}
Micromirrors used to steer optical beams at the tip of an endoscope are an active area of research. MEMS mirrors with various actuation mechanisms have been reported including rotating micromotors [1], parallel plate electrostatic [2], electrothermal $[3,4]$ and vertical electrostatic combs[5-8]. This paper presents for the first time a two-axis magnetically actuated micromirror designed for an OCT (Optical Coherence Tomography) endoscope. We demonstrate also for the first time a free-rotating axis with no spring constraint, combined with an orthogonal resonant drive axis. Magnetic actuation allows large angular deflections (+/- 40 degrees rotation) at low voltage $(<1 \mathrm{~V})$, a significant advantage relative to electrostatic mirrors for patient safety. Actuation is accomplished with 4 coils (two pairs) in proximity to the moving mirror, which contains a small permanent magnet. The entire endoscope scan engine is contained in a 2.8 $\mathrm{mm}$ ID plastic tube. Details of the endoscope design, MEMS fabrication, optical path, scanning, and sample OCT images are presented.
\end{abstract}

\section{INTRODUCTION}

Minimally invasive surgery is an important trend in medicine that leads to reduced trauma and faster patient recovery. Endoscopes are evolving rapidly to smaller sizes and higher functionality, including the ability to image tumors and in some cases treat diseased tissue with radiation or sound.

OCT (Optical Coherence Tomography) has recently evolved from the time-domain systems which sample 2,000 A-lines/sec to spectral domain, [9] and Optical Frequency Domain Imaging (OFDI) [13] which sample 19,000 to 100,000 A-lines/sec. In contrast, mechanically scanned endoscopes have not kept up with current acquisition speed capabilities of Spectral Domain OCT and Optical Frequency Domain Imaging (OFDI). Rotational scanners suffer non-uniform rotation distortion (NURD) from long, thin cables transmitting torque from the external motor. Linear scan mechanisms are limited to a few Hertz due to the forces needed to translate distally a fiber and lens assembly. Rapidly scanning MEMS micromirrors offer an alternative to mechanical actuation using external motors and mechanical linkages.

MEMS mirrors for endoscopes are an active area of research [1-8]. Arguably the most advanced of these use multi-level electrostatic comb actuators. These mirrors offer many advantages such as low mass, absence of exotic materials, and the possibility of built in capacitive feedback. Drawbacks include tiny gaps and high voltages which are potential failure modes and safety concerns. Previous work on magnetic actuation using a film of Permalloy on the mirror [10], a coil on the mirror [11] or screenprinted magnets [12] show that these can achieve large angular or linear scans at low voltages across large gaps, advantages in an endoscope. This work explores a novel design for a two-axis raster scan, using a resonant fast axis and free-rotating slow axis. A large angle raster scan is demonstrated. The entire system fits inside a $2.8 \mathrm{~mm}$ ID plastic tube. Preliminary 2-D images taken with both time-domain and spectral domain OCT are shown.

\section{THEORY}

OCT is an optical technique for 3D tissue imaging based on back-reflection from the sample. The back-reflected light is detected interferometrically by combining with light from a reference mirror. In time-domain OCT, the back-reflection at various depths is collected sequentially by moving the reference mirror. Newer techniques, such as spectral-domain OCT and OFDI, use Fourier techniques to transform an acquired spectrogram, and are an order of magnitude faster than timedomain OCT $[\mathbf{9}, \mathbf{1 3}]$.

The endoscope design is shown in Fig. 1. The MEMS mirror contains a small $\mathrm{NdFeB}$ permanent magnet $(0.6 \mathrm{~mm} \mathrm{X} 0.8 \mathrm{~mm} \mathrm{X}$ $0.2 \mathrm{~mm}$ ), and is actuated via two pairs of coils: a fast axis pair and a slow axis pair. FEA was used to calculate the torque $/ \mathrm{mA}$ along each axis (Fig. 3 and Table 1).

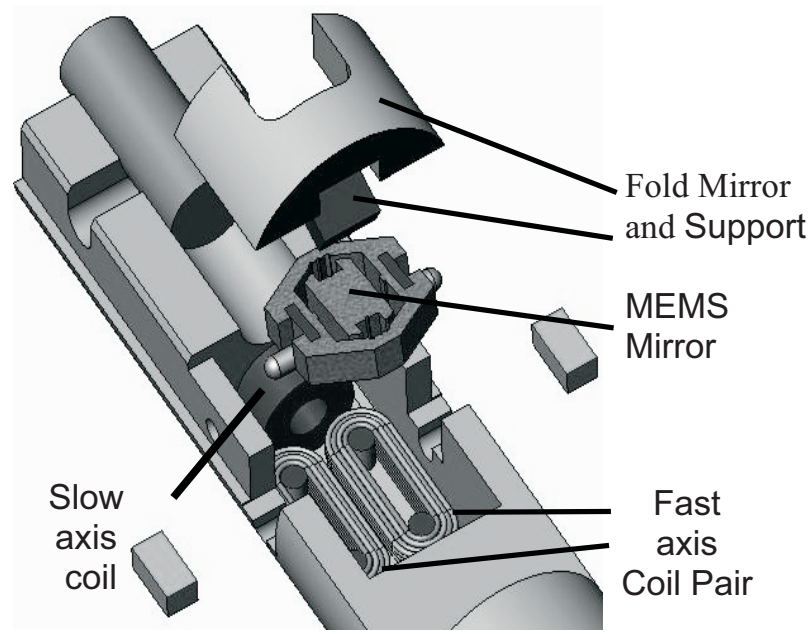

Figure 1. Solid model of endoscope system, showing actuation coils, fold mirror and MEMS mirror.

\section{Endoscope Optical Design}

Light from an SMF 28 optical fiber is focused through a GRIN lens, reflected from a fold mirror to the MEMS mirror which steers along two axes (Fig. 2). ZEMAX simulations were used to optimize the optical path for resolution over the 3dimensional imaging region. Maintaining good focus over large angular deflections is difficult due to cylindrical lensing effects from the plastic sheath of the endoscope. The light beam must avoid normal incidence on the plastic sheath, since this would create a strong back-reflection which would degrade the $\mathrm{S} / \mathrm{N}$ of 
spectral domain image processing. The fold mirror allows the MEMS mirror to steer the optical beam forward towards the endoscope tip.

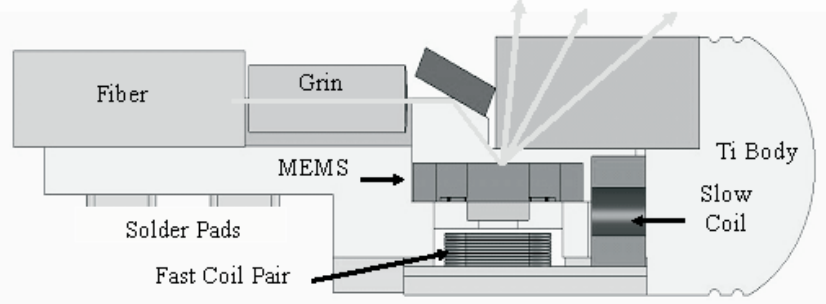

Figure 2. Solid model of later version of endoscope system, showing optical path through system and solder pads for strain relief. This version shows only one slow axis coil.

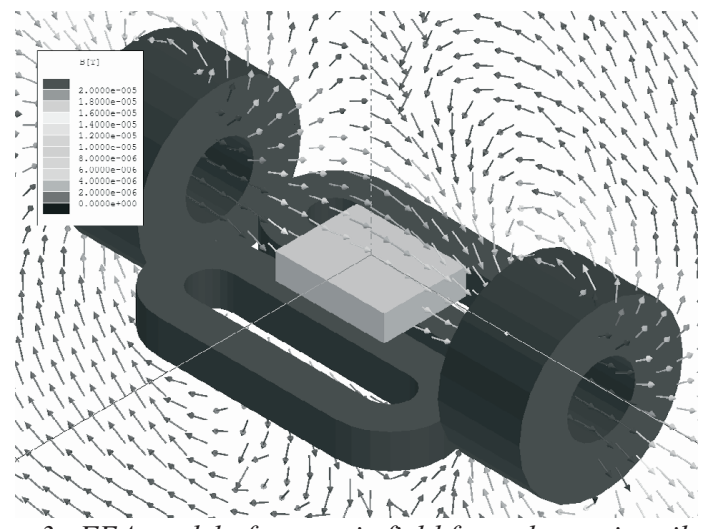

Figure 3. FEA model of magnetic field from slow-axis coils used to calculate torque/mA. Similar analysis used for fast axis coils.

These mirrors have a high Q (600-900) on the resonant axis, therefore driving them off-resonance requires care. For example, driving with a triangle wave is desirable for a raster scan. In this case, the input drive signal must be filtered with a notch or low pass filter to ensure that no high order harmonics are present at the resonant frequency, or ringing will result.

\section{EXPERIMENTAL DETAILS}

Fabrication Process: A simple, manufacturable process with only 3 photomask steps was used to fabricate the MEMS mirrors, illustrated in Fig. 4. Photoresist liftoff was used to pattern the metal mirror layer, consisting of $\mathrm{Cr} / \mathrm{Au}$ on the handle side of an SOI wafer.

The flexures and gimbal were etched using an STS ICP (inductively coupled plasma) etcher running the Bosch process. Folded flexures consisting of 3 beams were either 6 microns wide or 8 microns wide depending on design, and 50 microns thick (set by the device layer of the SOI wafer). Flexures, temporary etch buffers (used to create uniform etch conditions on both sides of each flexure leg) and stops are shown in Fig. 5.

A second ICP etch was used to pattern the handle side of the wafer (350 microns thick), freeing up the mirrors. A final HF etch was used to remove the buried oxide layer. Finally, temporary hold-downs on the SOI layer were snapped to release the mirrors from the wafer. Fig. 6 shows a completed device mirror-side up.

Magnets made from 50 MGOe $\mathrm{NdFeB}$ were attached individually using epoxy. Screen printed magnets have been demonstrated previously [12], but to achieve higher magnetic moment per unit mass, a solid rare-earth magnet was selected.
Axles for the slow axis rotation were also inserted manually and attached using epoxy (Figure 7). Axles were packed in Halocarbon stopcock grease for smooth rotation.

The endoscope body, fold mirror support, coil support and axles were machined from bio-compatible titanium using a combination of conventional machining and wire EDM.

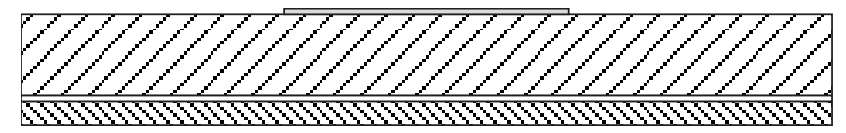

a. Metal 1 (Cr/Au) handle side lift-off,

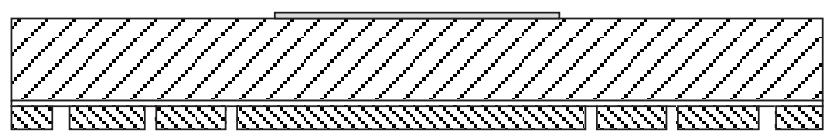

b. ICP etch 50 micron SOI layer to form springs and gimbal



c. ICP etch handle side to release mirror



d. HF etch to remove buried oxide.

Break temporary tabs to release mirrors

Figure 4. Fabrication sequence used to make mirror chips

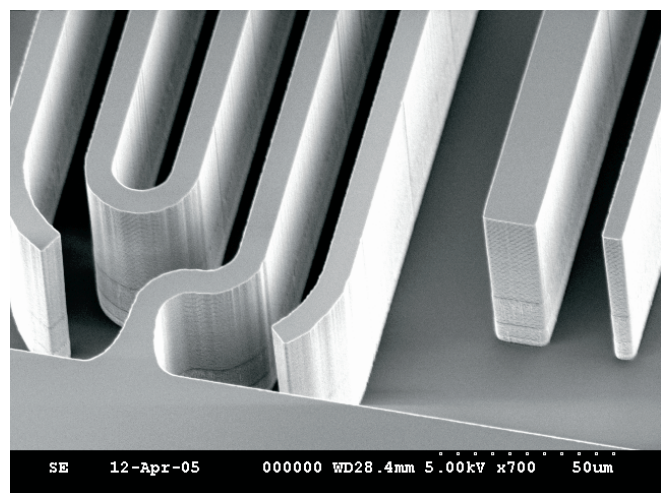

Figure 5. ICP etched folded flexure fabricated from single crystal silicon, shown with etch buffers and stops.

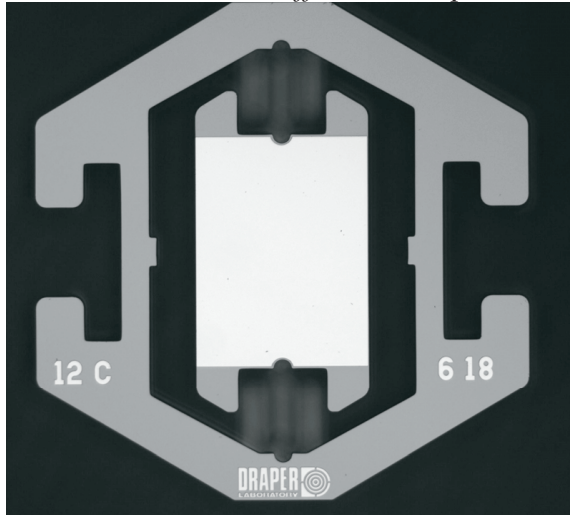

Figure 6. MEMS mirror photograph showing Au mirror and side notches (left and right side) for holding axles. 


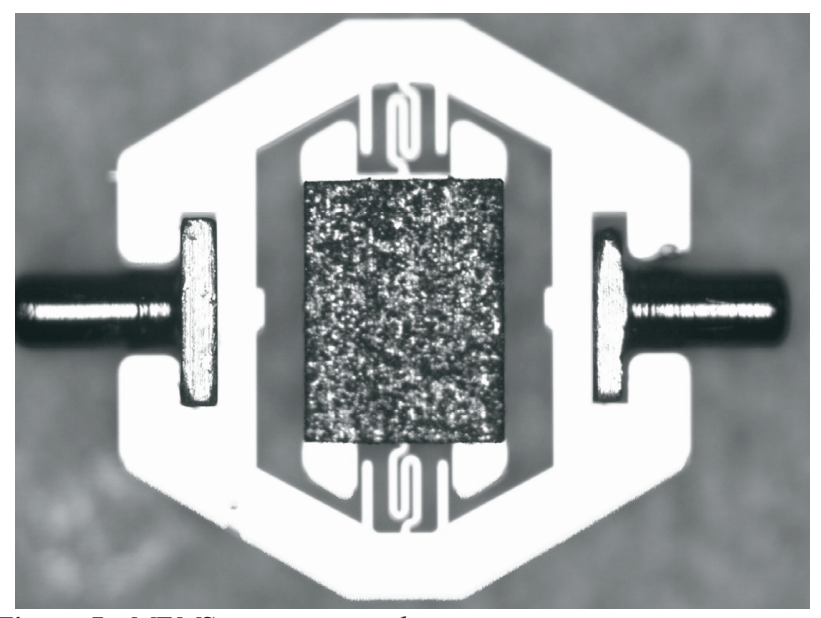

Figure 7. MEMS micromirror showing permanent magnet, springs, and axles.

\section{RESULTS}

A completed endoscope scanner is shown in Fig. 8. MEMS mirrors were fabricated with 6 micron and 8 micron wide flexures, giving resonant frequencies near $550 \mathrm{~Hz}$ and $800-900 \mathrm{~Hz}$ respectively. The measured $\mathrm{Q}$ of the fast axis is approximately 600-800, giving a large drive amplification at resonance.

Static angle vs. drive current data for a 6 micron flexure device is shown in Fig. 9. Optical deflection of $+/-8$ degrees was achieved at drive currents of $91 \mathrm{~mA}$ at $0.73 \mathrm{~V}$ drive voltage.

A linear scan at $10 \mathrm{~Hz}$ was achieved by driving the fast axis with a triangle wave (Fig. 10). Angle position of a laser spot reflected from the mirror was measured using a Hamamatsu Position Sensing Diode (PSD). Drive signals with harmonic content at the resonant frequency $(562 \mathrm{~Hz})$ produced ringing. This could be avoided by several input shaping techniques, such as notch filtering or low-pass filtering the drive signal.

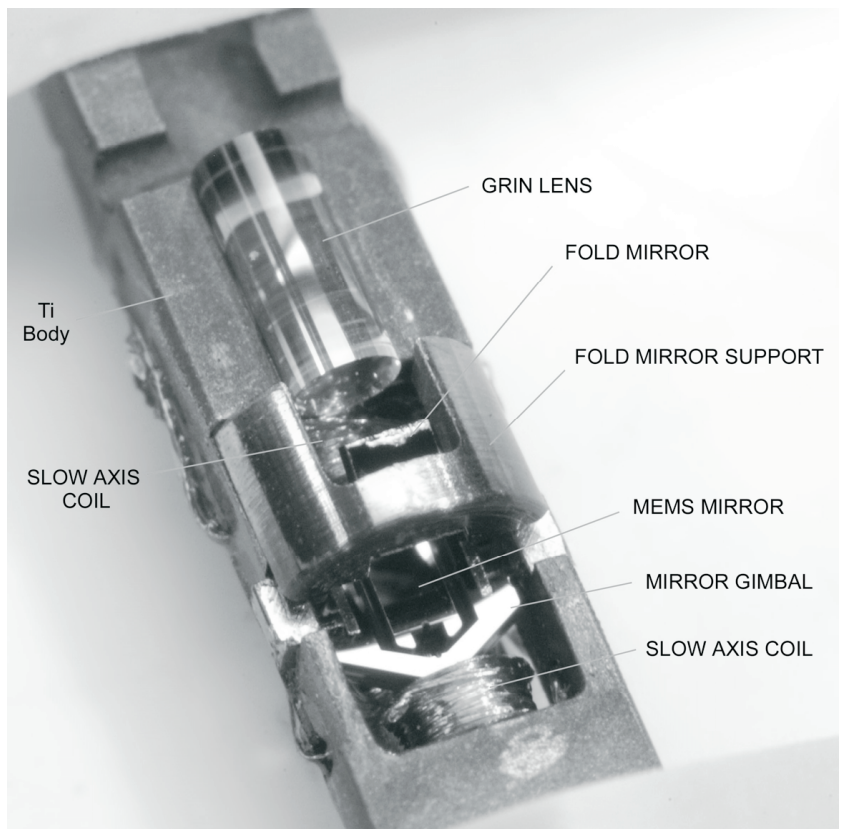

Figure 8. Completely assembled endoscope scan engine with actuation coils fits in a $2.8 \mathrm{~mm} I D$ tube.



Figure 9. Static fast axis angular deflection vs. drive current for mirror with 6 micron wide flexures.

Table 1. Coil Turns and Torque/A

\begin{tabular}{|c|c|c|}
\hline & Slow Axis & Fast Axis \\
\hline \# turns each coil & 300 & 30 \\
\hline Calc. Torque $(\mathrm{N}-\mathrm{m} / \mathrm{A})$ & $2.1 * 10^{-6}$ & $1.5 * 10^{-6}$ \\
\hline Coil Resistance $(\mathrm{ohms})$ & 58 & 8 \\
\hline
\end{tabular}



Figure 10. Mirror scan following $10 \mathrm{~Hz}$ triangle wave.

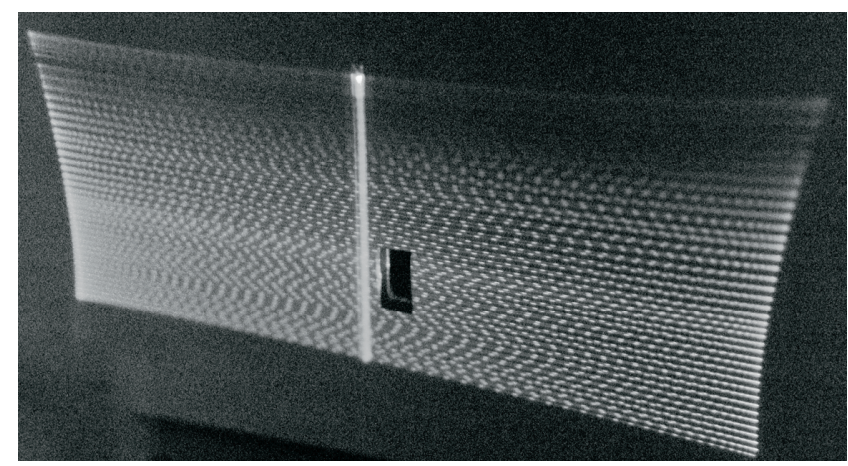

Figure 11. Two-axis raster scan produced by MEMS mirror. Laser passes through hole in screen to scanner, mounted on an optical bench. Mirror scans +/- 40 degrees optical both axes. 
Fig. 11 shows a combined fast resonant axis and orthogonal slow triangle wave scan. Fast axis scans were limited by the mirror hitting the gimbal at about $+/-44$ degrees optical. Slow axis axle supported scans were limited by the magnet touching the fast axis drive coils, also at $+/-40$ degrees depending on the magnet to coil gap.

OCT images: Fig. 12 shows a cross-sectional image of a researcher's finger using spectral domain OCT running at 18.5 frames/s. Fig. 13 shows an image of a finger tip using timedomain OCT at 1 frame/s.

The natural coordinate system for a rotating mirror is spherical, whereas Cartesian coordinates are preferred for imaging. We expect in the future software will be used for this coordinate conversion, and to store successive 2-D images as a 3-D data file.

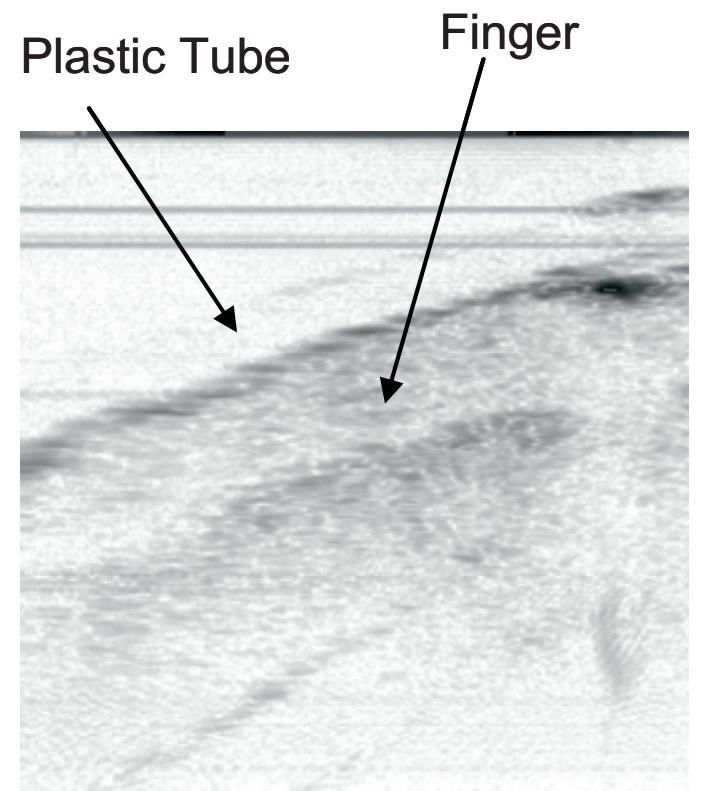

Figure 12. Spectral-domain OCT image using slow axis scan of MEMS endoscope.

\section{CONCLUSIONS}

We have designed and demonstrated an endoscope scan engine consisting of a 2-axis MEMS micro-mirror with an attached magnet driven by external copper coils. The resonant axis enables a large scan angle at low drive voltage. A slow axis supported by free-rotating axles was combined with the resonant drive axis to produce a 2-D raster scan. Preliminary OCT images from both Time-Domain OCT and Spectral-Domain OCT are shown.

\section{ACKNOWLEDGEMENTS}

The authors gratefully acknowledge the generous financial support for this research from CIMIT, the Center for the Integration of Medicine and Innovative Technology.

\section{REFERENCES}

1. P. Tran, D. Mukai, M. Brenner, and Z. Chen, "In vivo endoscopic optical coherence tomography by use of a rotational microelectromechanical system probe", Optics Letters, Vol. 29, No. 11, June 12004 pp. 1236-1238.

2. J. Yeow, X. Yang, A. Chahwan, M. Gordon, B. Qi, I. Vitkin, B. Wilson and A. Goldenberg, "Micromachined 2-D scanner for 3-D optical coherence tomography", Sensors and Actuators A 117(2005) pp. 331-340.

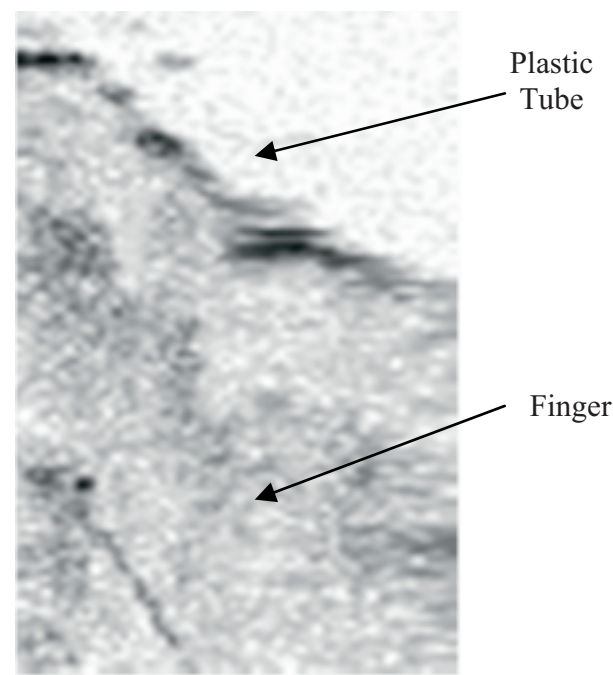

Figure 13. Time-domain OCT image using slow axis scan of MEMS endoscope.

3. A Jain, A. Kopa, Y. Pan, G. Fedder, and H. Xie, “A Two Axis Electrothermal Micromirror for Endoscopic Optical Coherence Tomography, IEEE Journal of Selected Topics in Quantum Electronics, Vol. 10, N. 3, May/June 2004 pp. 636-642.

4. T. Xie, H. Xie, G. Fedder and Y. Pan, "Endoscopic optical coherence tomography with new MEMS mirror", Electronics Letters, 16 October 2003, Vol. 39 No. 21, online \#20030998.

5. L. Fan, W. Piyawttanametha, M. Wu, A. Aguirre, P. Herz, Y.

Chen, and J. Fujimoto, "High Resolution 3D OCT Imaging with a

MEMS Scanning Endoscope", MOEMS and Miniaturized Systems

V, Proceedings of SPIE Vol. 5719, 2005 pp. 140-143.

6. W. Jung, D. McCormick, J. Zhang, N. Tien, and Z. Chen, Optical coherence tomography based on high-speed scanning MEMS mirror, Proceedings of SPIE Volume 5690, April 2005, pp. 342-348.

7. V. Milanovic, G. Matus and D. McCormick, "Gimbal-Less Monolithic Silicon Actuators for Tip-Tilt-Piston Micromirror Applications, IEEE Journal of Selected Topics in Quantum Electronics, Vol. 10, No. 3, May/June 2004, pp. 462-471.

8. V. Milanovic, Multilevel Beam SOI MEMS Fabrication and Applications, Journal of Microelectromechanical Systems, Vol. 13, No. 1, February 2004, pp. 19-29.

9. S. H. Yun, G. J. Tearney, B. E. Bouma, B. H. Park, and J. F. de Boer, "High-speed spectral-domain optical coherence tomography at $1.3 \mathrm{~mm}$ wavelength." Optics Express 11 (26), 3598-3604 (2003).

10. J.W. Judy and R.S. Muller, "Magnetically Actuated, Addressable Microstructures" IEEE JMEMS, vol. 6, no. 3, September 1997, pp. 249-256.

11. J. J. Bernstein, W. P. Taylor, J. D. Brazzle, C. J. Corcoran, G. Kirkos, J. E. Odhner, A. Pareek, M. Waelti, and M. Zai, "Electromagnetically Actuated Mirror Arrays for use in 3-D Optical Switching Applications", IEEE JMEMS, Vol. 13. No. 3, June 2004, pp 526-535.

12. L.K. LaGorce, O. Brand, and M.G. Allen, "Magnetic Microactuators Based on Polymer Magnets", IEEE JMEMS, Vol. 8, No. 1, March 1999, pp. 2-8.

13. W. Y. Oh, S. H. Yun, B. J. Vakoc, G. J. Tearney, and B. E. Bouma "Ultrahigh-speed optical frequency domain imaging and application to laser ablation monitoring" APPLIED PHYSICS LETTERS 88, 103902, 2006. 